\title{
To Assess Association of Bullous Pemphigoid and Neurological Disorders- A Clinical Study
}

\author{
Balkrishna Pralhadrao Nikam ${ }^{1}$, Dhanraj D Chavan ${ }^{2}$, Nischhal Shrivastava ${ }^{2}$ \\ ${ }^{1}$ Assistant Professor, ${ }^{2}$ PG Resident, Department of Dermatology, Krishna Institute of Medical Sciences Deemed to \\ be University, Karad
}

\begin{abstract}
Background: Bullous pemphigoid is an immunobullous disease which affects the elderly. The present study was conducted to assess association of Bullous pemphigoid and neurological disorders.

Materials \& Method: The present study was conducted on 125 cases of pemphigoid. Parameters such as name, age, gender, clinical features, etc were noted. The medical history, neurological diseases and medications used by the patients were recorded.

Results: Out of 125 cases, 65 were in males and 60 were in females. Common neurological disorders were dementia seen in 15, stroke in 12, Hemorrhagic stroke in 6, Parkinson's disease in 14 and epilepsy in 5. The difference was significant $(\mathrm{P}<0.05)$. There was positive correlation of dementia, stroke and Parkinson's disease with Bullous pemphigoid $(\mathrm{P}<0.05)$.
\end{abstract}

Conclusion: Authors found that positive correlation of dementia, stroke and Parkinson's disease with Bullous pemphigoid.

Key words: Bullous pemphigoid, Dementia, Parkinson's disease.

\section{Introduction}

Bullous pemphigoid is an immunobullous disease which affects the elderly. The incidence of BP has been estimated to be 4.5 to 14 new cases per million per year. The prevalence is shown to be increasing in some studies. ${ }^{1}$

Bullous pemphigoid (BP) is an autoimmune subepidermal blistering skin disorder which occurs mostly in the elderly. ${ }^{2}$ It is associated with circulating autoantibodies against hemidesmosomal proteins BP180 (BPAG2) and BP230 (BPAG1) in the dermoepidermal junction. Clinical features of this disease include subepidermal blisters on urticarial plaques, erythematous, or noninflamed skin. It occurs mostly on the flexural aspects of the limbs and on the trunk. BPAG2 is a transmembrane protein that has a long extracellular domain and is associated with synapse stabilization in the central nervous system. BPAG1 is an intracellular protein which belongs to the plakin family. ${ }^{3}$
The role of BPAG1 is to connect the intermediate filament, microtubule, and microfilament cytoskeletal networks with each other and to cell membrane sites. They are also known as scaffolds for signaling proteins that modulate cytoskeletal dynamics. Different isoforms of BPAG1 have been found: BPAGI-e in the skin, BPAG1-a in the nervous system, and BPAG1-b in the striated muscle. $^{4}$

There is growing evidence to suggest an association between BP and a range of neurological disorders including dementia, cerebral stroke, Parkinson's disease, cerebrovascular disease, multiple sclerosis, epilepsy, and polyneuropathies. While the immunological mechanisms have not been fully elucidated the association between $\mathrm{BP}$ and neurological disorders may be due to immune responses arising from cross-reactivities of skin auto antibodies with BP antigens or their isoforms that are known to be expressed in brain and neuronal tissue. ${ }^{5}$ The present study was conducted to assess association of Bullous pemphigoid and neurological disorders. 


\section{Materials \& Method}

The present study was conducted in the department of Oral Medicine \& Radiology. It comprised of 125 cases of pemphigoid. Ethical clearance was obtained from institutional ethical committee. All patients were informed regarding the study and written consent was obtained.

Parameters such as name, age, gender, clinical features, etc were noted. The medical history, neurological diseases and medications used by the patients were recorded. Data thus obtained were subjected to statistical analysis. $\mathrm{P}$ value less than 0.05 was considered significant.

\section{Results}

Table I Distribution of cases

\begin{tabular}{|c|c|c|}
\hline \multicolumn{3}{|c|}{ Total- 125 } \\
\hline Gender & Males & Females \\
\hline Number & 65 & 60 \\
\hline
\end{tabular}

Table I shows that out of 125 cases, 65 were in males and 60 were in females.

Table II Neurological disorders

\begin{tabular}{|l|l|l|}
\hline $\begin{array}{l}\text { Neurological } \\
\text { disorders }\end{array}$ & Number & \multirow{2}{*}{ P value } \\
\hline Dementia & 15 & \multirow{2}{*}{0.05} \\
\cline { 1 - 2 } Stroke & 12 & \\
\cline { 1 - 2 } $\begin{array}{l}\text { Hemorrhagic } \\
\text { stroke }\end{array}$ & 6 & \\
\cline { 1 - 1 } $\begin{array}{l}\text { Parkinson's } \\
\text { disease }\end{array}$ & 14 & \\
\cline { 1 - 2 } Epilepsy & 5 & \\
\hline
\end{tabular}

Table II shows that common neurological disorders were dementia seen in 15 , stroke in 12 , Hemorrhagic stroke in 6, Parkinson's disease in 14 and epilepsy in 5. The difference was significant $(\mathrm{P}<0.05)$.
Table III Correlation of Neurological disorders and Bullous pemphigoid

\begin{tabular}{|l|l|l|}
\hline $\begin{array}{l}\text { Neurological } \\
\text { disorders }\end{array}$ & R value & P value \\
\hline Dementia & 0.321 & 0.04 \\
\hline Stroke & 0.314 & 0.01 \\
\hline $\begin{array}{l}\text { Hemorrhagic } \\
\text { stroke }\end{array}$ & 0.982 & 0.12 \\
\hline $\begin{array}{l}\text { Parkinson's } \\
\text { disease }\end{array}$ & 0.412 & 0.05 \\
\hline Epilepsy & 0.824 & 0.54 \\
\hline
\end{tabular}

Table III shows that there was positive correlation of dementia, stroke and Parkinson's disease with Bullous pemphigoid $(\mathrm{P}<0.05)$.

\section{Discussion}

Several mechanisms have been postulated to explain the association between bullous pemphigoid and neurological disorders. Firstly, in terms of immunology and inflammation, neuronal isoforms of BP180 and 230 antigens lead to cross reactivity between the skin and the brain. ${ }^{6}$ This process is further facilitated by damage to the blood-brain barrier due to the neurological disorder. Systemic inflammation involving the $\mathrm{T}$ helper 1 and $\mathrm{T}$ helper 2 immune responses has also been found in bullous pemphigoid as evidenced by elevated serum levels of cytokines. ${ }^{7}$

Clinical manifestations include pruritus and urticated, erythematous lesions which later develop into large, tense subepidermal blisters and mucosal involvement. An association of bullous pemphigoid with neurological diseases such as stroke, dementia, Parkinson's disease, epilepsy, amyotrophic lateral sclerosis, syringomyelia and multiple sclerosis has been reported. ${ }^{8}$ The present study was conducted to assess association of Bullous pemphigoid and neurological disorders.

In this study out of 125 cases, 65 were in males and 60 were in females. Chen et $\mathrm{al}^{9}$ conducted a retrospective case-control study involving 43 patients with bullous pemphigoid and 43 age-, sex- and ethnicity-matched controls. There was a statistically significant association between bullous pemphigoid and neurological disorders 
in particular for dementia. Although stroke was more common among patients with bullous pemphigoid, this association was not statistically significant with OR of 1.9 and adjusted OR of 2.1. Similarly both ischaemic stroke and hemorrhagic stroke were more common. Other neurological disorders more common among patients with bullous pemphigoid were Parkinson's disease and epilepsy. Dyslipidaemia was significantly less common among patients with bullous pemphigoid.

We found that common neurological disorders were dementia seen in 15 , stroke in 12 , Hemorrhagic stroke in 6, Parkinson's disease in 14 and epilepsy in 5. Yang et al ${ }^{10}$ in their cross-sectional study, 87 patients with BP were enrolled. Out of 87 patients with BP, 17 (19.5\%) had at least one neurological disease. Cerebrovascular accident (CVA) was the most common neurological disease that was seen in 7 patients $(8.0 \%)$ in the case group and $4(2.1 \%)$ in the control group. The incidence of CVA was significantly different between BP patients and the control group $(P=0.022)$. Dementia was observed in 6 patients in the case group $(16.8 \%)$ and 2 $(1.0 \%)$ in the control group. The incidence of dementia was significantly different between BP patients and the control group $(P=0.008)$. In this study, the incidences of Parkinson's disease $(P=0.830)$, epilepsy $(P=0.067)$, and multiple sclerosis $(P=0.326)$ were not statistically significant between the two groups.

We found a positive correlation of dementia, stroke and Parkinson's disease with Bullous pemphigoid $(\mathrm{P}<0.05)$. Langan et $\mathrm{al}^{11}$ retrospectively assessed 183 patients with BP and 348 age- and sex-matched controls for neurological disorders. Overall, there was a highly statistically significant association between BP and neurological disorders. These included dementia, Parkinson's disease, stroke and other neurological disorders but not Alzheimer's diseases, which was more common among patients in the control group.

Texeira et a $1^{12}$ observed that neurological disorders which cause central nervous system inflammation or degeneration were related to BP and the association was strongest between multiple sclerosis and BP (13). Indeed, an association between multiple sclerosis and BP has also been observed in several other reports. Interestingly, other conditions such as dementia, Parkinson's disease, epilepsy, stroke, schizophrenia, schizotypal and delusional disorders, as well as personality disorders also revealed a statistically significant association with BP.

\section{Conclusion}

Authors found that positive correlation of dementia, stroke and Parkinson's disease with Bullous pemphigoid.

Ethical Clearance- Taken from Institutional Ethical committee

\section{Source of Funding- Self}

Conflict of Interest- Nil

\section{References}

1. Jung M, Kippes W, Messer G, Zillikens D, Rzany B. Increased risk of bullous pemphigoid in male and very old patients: A population-based study on incidence. J Am Acad Dermatol. 1999;41:266-8.

2. Stanley JR, Hawley-Nelson P, Yuspa SH, Shevach EM, Katz SI. Characterization of bullous pemphigoid antigen: A unique basement membrane protein of stratified squamous epithelia. Cell. 1981;24:897-903.

3. Diaz LA, Ratrie H, 3rd, Saunders WS, et al. Isolation of a human epidermal cDNA corresponding to the $180-\mathrm{kD}$ autoantigen recognized by bullous pemphigoid and herpes gestationis sera. Immunolocalization of this protein to the hemidesmosome. J Clin Invest. 1990;86:1088-94.

4. Stanley JR. Cell adhesion molecules as targets of autoantibodies in pemphigus and pemphigoid, bullous diseases due to defective epidermal cell adhesion. Adv Immunol. 1993;53:291-325.

5. Zhang LM, Wu J, Xiao T, et al. Treatment and mortality rate of bullous pemphigoid in China: a hospital-based study. Eur J Dermatol 2013; 23(1): 94-8.

6. Kulthanan K, Chularojanamontri L, Tuchinda P, et al. Prevalence and clinical features of Thai patients with bullous pemphigoid. Asian Pac J Allergy Immunol 2011; 29(1): 66-72.

7. Cai SCS, Allen JC, Lim YL, et al. Mortality of bullous pemphigoid in Singapore: risk factors and causes of death in 359 patients seen at the National Skin Centre. Brit J Dermatol 2014; 170(6): 131926.

8. Taghipour K, Chi CC, Vincent A, et al. The Association of Bullous Pemphigoid with Cerebrovascular Disease and Dementia. Arch Dermatol 2010; 146(11): 1251-4. 
9. Chen YJ, Wu CY, Lin MW, et al. Comorbidity profiles among patients with bullous pemphigoid: a nationwide population-based study. Brit J Dermatol 2011; 165(3): 593-9.

10. Yang YW, Chen YH, Xirasagar, S et al. Increased Risk of Stroke in Patients with Bullous Pemphigoid: A Population-Based Follow-Up Study. Stroke 2011; 42(2): 319-23.
11. Langan SM, Groves RW, West J. The relationship between neurological disease and bullous pemphigoid: A population-based case-control study. J Invest Dermatol 2011; 131(3): 631-6.

12. Texeira VB, Cabral R, Brites MM, et al. Bullous pemphigoid and comorbidities: A case-control study in Portuguese patients. An Bras Dermatol 2014; 89(2): 274-8. 